\title{
La violencia feminicida en el Estado de Guanajuato. Feminicidios, impunidad y tradición ${ }^{1}$
}

\author{
Rocío Rosas Vargas ${ }^{2}$
}

\author{
Fecha de recepción: 27 de noviembre de 2017 \\ Fecha de aprobación: 16 de abril de 2018
}

\begin{abstract}
Resumen
En el estado de Guanajuato, México, la violencia de género y los feminicidios presentan cifras alarmantes y en aumento. Si bien este año aún no termina, los asesinatos de mujeres son mayores que los de 2016, en muchos de los cuales no hay un asesino claramente identificado. Este fenómeno se suma al incremento de la violencia hacia las mujeres, en el que $60 \%$ de ellas dijeron haber padecido algún tipo de violencia al menos una vez en su vida (Endireh, 2016). El objetivo de este trabajo se centra en analizar la violencia de género y feminicida y sus implicaciones y consecuencias en la vida de las mujeres, y explicar la relación entre el aumento de la violencia de género y los feminicidios; ambos fenómenos resultan impunes porque los roles tradicionales de género refuerzan el sistema patriarcal que hace que las mujeres sigan subordinadas a lo masculino, y que aquellas que parecen apartarse de esos roles tradicionales sean castigadas con violencia que puede escalar a los feminicidios. Lo anterior porque es en la zona llamada "Corredor Industrial del Estado" donde más feminicidios se han perpetrado, pero ninguna zona, ningún municipio del Estado está exento de este fenómeno.
\end{abstract}

Palabras clave: Derechos humanos, feminicidios, Guanajuato, México, violencia de género.

\section{Femicide violence in the State of Guanajuato. Feminicides, impunity and tradition}

\begin{abstract}
In the state of Guanajuato, Mexico, gender violence and femicides present alarming and increasing numbers. While this year has not yet ended the
\end{abstract}

Este artículo se deriva del proyecto de investigación "Feminicidios en el Estado de Guanajuato," que está en proceso y es financiado por la Universidad de Guanajuato. Agradezco el apoyo de Sara Josefina Ibarra Montenegro, estudiante de la Licenciatura en Desarrollo Regional, en el trabajo de recopilación de notas para este artículo.

2 Doctora en Estudios del Desarrollo Rural. Profesora de Tiempo Completo de la Universidad de Guanajuato. Salvatierra, Guanajuato, México. Contacto: atximba@yahoo.com.mx y rociorv@ugto.mx. 
murders of women are greater than those of 2016, in many of which there is no killer clearly identified. This phenomenon adds to the increase in violence against women, where $60 \%$ of them said they had suffered some type of violence at least once in their lives (Endireh, 2016). Therefore, the objective of this work is to analyze gender violence and feminicide, its implications and consequences in women's lives and explain the relationship between the increase in gender violence and femicides, both phenomena are unpunished that traditional gender roles reinforce the patriarchal system that makes women remain subordinated to the masculine, and that those that seem to deviate from those traditional roles are punished with violence that can escalate to femicides. The above is affirmed because it is in the area called the State Industrial Corridor where more feminicides have been perpetrated, but no area, no municipality of the state is exempt from this phenomenon.

Keywords: Human rights, feminicides, Guanajuato, Mexico, gender violence.

\title{
A violência feminicida no Estado de Guanajuato. Feminicídios, impunidade e tradição
}

\begin{abstract}
Resumo
No estado de Guanajuato, México, a violência de gênero e os feminicídios apresentam números crescentes e alarmantes. Só neste ano que ainda não acaba, os assassinatos de mulheres têm sido mais do que os de 2016, em muitos dos quais não há um assassino claramente identificado. Este fenômeno soma-se ao incremento da violência contra as mulheres, no que $60 \%$ delas disseram ter padecido algum tipo de violência pelo menos uma vez na sua vida (Endireh, 2016). O objetivo deste trabalho centrase em analisar a violência de gênero e feminicida e as suas implicações e consequências na vida das mulheres, e explicar a relação entre o aumento da violência de gênero y os feminicídios; ambos os fenômenos ficam impunes porque os papéis tradicionais de gênero reforçam o sistema patriarcal que faz com que as mulheres sigam subordinadas ao masculino, e que aquelas que parecem afastar-se desses papéis tradicionais sejam castigadas com violência que pode escalar aos feminicídios. $\mathrm{O}$ anterior porque é na zona chamada de "Corredor Industrial do Estado" onde mais feminicídios têm se perpetrado, mas nenhuma zona, nenhuma prefeitura do Estado fica isenta deste fenômeno.
\end{abstract}

Palavras-chave: Direitos humanos, feminicídios, Guanajuato, México, violência de gênero.

\section{Introducción}

En 2007 se aprobó en México la Ley General de Acceso de las Mujeres a una vida libre de violencia, en la cual se define la violencia de género como "Cualquier acción u omisión, basada en su género, que les cause daño o sufrimiento psicológico, físico, patrimonial, económico, sexual o la muerte, tanto en el ámbito 
privado como en el público" (p. 11). Esta ley posteriormente fue aprobada para cada Estado en el país, y Guanajuato fue uno de los últimos Estados donde se aprobó, ya que la emitió en noviembre de 2010 .

La Ley define la violencia feminicida como "la forma extrema de violencia de género contra las mujeres, producto de la violación de sus derechos humanos, en los ámbitos público y privado, conformada por el conjunto de conductas misóginas que pueden conllevar impunidad social y del Estado y puede culminar en homicidio y otras formas de muerte violenta de mujeres; y cualquier otra forma análoga que lesione o sea susceptible de dañar la dignidad, integridad o libertad de las mujeres" (Pérez, 2010, pp. 71-72).

Huacuz (2011, citando a Heise, 1994) afirma que la violencia hacia las mujeres puede entenderse como un acto de fuerza física o verbal, coercitiva o privación amenazadora para la vida, dirigida a mujeres o niñas, que cause daño físico o psicológico, humillación o privación arbitraria de la libertad, y que perpetúe la subordinación femenina. En esta definición, afirma Huacuz, se distingue claramente el sujeto a quien va dirigida la violencia, los tipos de acciones, los tipos de daños y la pretensión última de la violencia: perpetuar la subordinación de las mujeres.

El concepto de violencia de género pone en evidencia el carácter estructural de la violencia al denunciar la existencia de un marco patriarcal de las relaciones que hace de mujeres y hombres, de las unas y de los otros, lo que somos, y que nos impulsa a hacer lo que hacemos. (Izquierdo, 2008, citada en Huacuz, 2011, p. 19)

En las sociedades patriarcales y autoritarias las mujeres pueden padecer episodios crecientes de violencia, que incluso pongan en riesgo sus vidas; la vulnerabilidad de mujeres y muchachas puede ser mayor debido al contexto en el que viven, es decir, "caracterizado por la ignorancia, la pobreza y el aislamiento; la predominancia de sistemas patriarcales que norman despótica- 
mente las relaciones matrimoniales, la ausencia de figura paterna para brindarle algún tipo de protección, la presencia de una madre que le impone una relación degradante, su transformación en una víctima permanente a merced de un marido golpeador, el desprecio y la desatención que acaba en su muerte y que ni los hijos, ya grandes, pudieron detener" (Marroni, 2004, pp. 210-211).

Para analizar los casos de violencia de género y feminicidios que se tratarán en este artículo, a continuación se presentan algunos conceptos que pudieran ayudarnos a entender estos delitos.

Siguiendo el análisis de los conceptos de violencia de género, Ramos y Saltijeral (2011) hablan de "terrorismo íntimo", en el caso de las mujeres violentadas (e incluso asesinadas) por sus parejas varones. En su estudio realizan una caracterización de las mujeres que padecen o padecieron "terrorismo íntimo": se casaron con hombres mucho mayores que ellas, mínimo cinco años mayores; tienen más hijos (más de cuatro) que otras que no padecen terrorismo íntimo; carecen de apoyo para las labores domésticas, pues sus esposos nunca realizan este tipo de trabajo; tienen menor escolaridad; más del $50 \%$ provee el sustento a sus familias, por lo que sus parejas son las que menos aportan a la familia; los hombres de estas mujeres ganan menos que otros; sus esposos son alcohólicos. Como afirma Izquierdo (2011), los esposos de estas mujeres son despojados en el ámbito patriarcal, se sienten disminuidos y quizás por eso violentan a sus mujeres. Este concepto podría aplicarse a algunos casos de feminicidios, sobre todo a los perpetrados por las parejas o exparejas, y tal vez aplicarse a los casos ocurridos en la zona llamada "corredor industrial", donde en 2017 ha ocurrido la mayoría de los casos de feminicidios.

"Terrorismo patriarcal", entonces, se define como " $[\mathrm{u}] \mathrm{n}$ producto de las tradiciones patriarcales del derecho de los hombres de controlar a 'sus' mujeres, es una forma de control terrorista de las esposas por sus maridos e implica el uso sistemático, no sólo de la violencia, sino de la subordinación económica, amenazas, 
aislamiento, y otras tácticas de control" (Johnson, 1995, p. 284, citado en Izquierdo, 2011, p. 35). De igual forma, este concepto podría utilizarse para el análisis de algunos de los casos de feminicidios en el Estado.

Cruz (2011) utiliza el término "terrorismo fálico", que define como no solo el control y dominio absoluto sobre las mujeres, "sino demostrar este poder mediante la penetración, la posesión y el goce sexual del cuerpo femenino para su uso y desecho" (p. 168). Adopta este concepto para analizar los feminicidios en Ciudad Juárez, donde hay una relación clara entre estos asesinatos de poder y el goce masculino sobre un cuerpo indefenso femenino, y no sabemos hasta dónde este concepto se aplique a algunos de los feminicidios ocurridos en el Estado de Guanajuato

\section{Definiendo al feminicidio}

El feminicidio se define, según Arteaga (2010, p. 6), como "no sólo el asesinato de una mujer, sino como la desarticulación de todas sus posibilidades de resistencia mediante el empleo de formas de degradación psicológica, tortura o violación".

El Código Penal del Estado de Guanajuato, en su última actualización (julio, 2016, p. 32-33), define el feminicidio como sigue:

ARTÍCULO 153-a.- Habrá feminicidio cuando la víctima del homicidio sea mujer y la privación de la vida se cometa por razones de género, considerándose que existen éstas, cuando ocurra alguno de los siguientes supuestos en agravio de la víctima:

I. Que haya sido incomunicada;

II. Que haya sido violentada sexualmente;

III. Que haya sido vejada;

IV. Que se le hayan infligido lesiones o mutilaciones, infamantes o degradantes aún respecto del cadáver; 
V. Que haya existido amenazas, acoso, lesiones o violencia en el ámbito familiar, laboral o escolar, o cualquier otro que implique supra o subordinación del sujeto activo en contra de ella;

VI. Que exista o haya existido con el activo relación íntima, de convivencia, de confianza, noviazgo, parentesco, matrimonio o concubinato; o

VII. Que su cuerpo sea expuesto o arrojado en un lugar público.

Esta definición considera la presencia de uno o varios de los supuestos para ser considerado feminicidio: no dice que se deban cumplir todos. Con lo cual el número de feminicidios en el Estado es mayor que lo que la misma autoridad estatal afirma.

Aun cuando el feminicidio esté definido jurídicamente, creemos que debemos partir de otro tipo de análisis; en este caso partimos del sociológico y feminista, sin olvidar por supuesto lo que dice el Código mencionado. Pero además, como afirma Bodelón (2009), la estructura jurídica debe pensarse como parte de las relaciones sociales. Porque, por ejemplo, no se consideraría feminicidio si en un ajuste de cuentas matan a una mujer, pareja de uno de los contendientes, porque no se aplicaría ninguno de los supuestos arriba mencionados. Pero desde las estructuras sociales analizadas con la óptica del feminismo, consideramos que la mujer en cuestión podría haber tenido una relación de subordinación con su pareja y, bajo esa relación, haber acompañado o participado en los delitos y con ello puede tratarse de un feminicidio. Porque hay un sesgo de género en las relaciones, tanto sociales, comerciales, como personales. De ahí que "[c]uando se concentran o aumentan las formas de opresión y violencia, las mujeres quedan en vulnerabilidad y, en el extremo, en riesgo de ser asesinadas. La base de los crímenes contra las mujeres radica en esas condiciones de vida" (Lagarde, 2006, p. 28).

Lagarde se refiere al feminicidio como "el conjunto de delitos de lesa humanidad que contienen los crímenes, los secuestros y 
las desapariciones de niñas y mujeres en un cuadro de colapso institucional. Se trata de una fractura del estado de derecho que favorece la impunidad. El feminicidio es un crimen de estado" (2006, p. 30). Concordamos con ella cuando afirma que es la parte más visible de la violencia hacia las mujeres, pero creemos que, si hay aumento de estos crímenes, la violencia de género va también en franco ascenso. En el Estado de Guanajuato seis de cada diez mujeres afirmaron haber pasado por algún episodio de violencia a lo largo de su vida, y es un dato que aumentó comparado con años anteriores (Endireh, 2016). También pensamos que, debido al aumento de la violencia en general en el Estado, sobre todo por obra del crimen organizado, los feminicidios aumentarán aún más y muchos de ellos quedarán impunes. Al momento de corregir este artículo, los asesinatos de mujeres ya suman más de 80 (mayo de 2018), por lo que esta proyección se está cumpliendo, pues muchos de los casos pareciera ser que se trata de crímenes relacionados con la delincuencia organizada.

Russell (2006, p. 74) define al feminicidio como "el asesinato de mujeres por hombres por ser mujeres". Estos asesinatos, a decir de la autora, son motivados por el odio, el desprecio, el placer o un sentido de propiedad hacia las mujeres. Cuando algunos hombres creen que su autoridad ha sido desafiada se sienten con derecho a matar a las mujeres que supuestamente los desafían; otros porque no toleran el rechazo del cual se sienten objetos. La misma Russell afirma que el feminicidio "es una forma de terrorismo que funciona para definir las líneas de género, para promulgar y reforzar el dominio masculino y para volver a todas las mujeres crónica y profundamente inseguras" (Russell, 2006, p. 346). Esta misma autora habla de varios tipos de feminicidios, como el íntimo, que parece ser el más abordado. Pero también se refiere al feminicidio social o encubierto, el cual se genera por las estructuras sociales misóginas existentes, que no reconocen los derechos de las mujeres y que tienen como consecuencia la muerte de ellas, por ejemplo las producidas por abortos mal practicados. 
Rita Segato, cita a Caputi y Russell cuando definen que El feminicidio representa el extremo de un continuum de terror anti-femenino e incluye una amplia variedad de abusos verbales y físicos tales como violación, tortura, esclavitud sexual (particularmente por prostitución), abuso sexual infantil incestuoso o extra-familiar, golpizas físicas y emocionales, acoso sexual (por teléfono, en las calles, en la oficina, y en el aula), mutilación genital (clitoridectomías, escisión, infubilaciones), operaciones ginecológicas desnecesarias (histerectomías gratuitas), heterosexualidad forzada, esterilización forzada, maternidad forzada (por la criminalización de la contracepción y del aborto), psicocirugía, negación de comida para las mujeres en algunas culturas, cirugía plástica, y otras mutilaciones en nombre del embellecimiento. Siempre que estas formas de terrorismo resulten en muerte, ellas se transforman en feminicidios (Caputi y Russell, 1992, p. 15, citado en Segato, 2006, p. 3).

El ambiente en el que se combina el poder y la masculinidad se impregna de odio hacia las mujeres y origina los feminicidios y su justificación. Se desatan por el deseo de controlar los cuerpos femeninos o porque ellas desafían el desequilibrio de poder existente en las sociedades, ya que acceden a mayores posiciones económicas y de poder, por lo que, para Segato (2006), estos crímenes son claramente crímenes de poder y de reducción de lo femenino. Ella misma afirma que "estos crímenes se adaptan de forma perfecta a un contexto faccional, en el eje horizontal de pares en la masculinidad representa también al eje de la hermandad corporativa involucrada en los negocios ilegales de la región" (Segato, 2006, p. 7). Los principales interlocutores de estos crímenes son los otros hombres, es un discurso que se dirige hacia sus pares en una demostración de poder hacia las víctimas. Tal vez sea por eso que, al menos en México, los hombres son más reacios a aceptar el término "feminicidio"; lo llaman "crimen pa- 
sional", en el mejor de los casos, y tienden a culpar a las víctimas de ser asesinadas.

Para Segato (2006), es de suma importancia tipificar los diferentes feminicidios, ya que quizás ello pudiera aportar datos precisos para la identificación de los asesinos, y que las leyes hagan categorías más precisas, pues algunos podrían no considerarse feminicidios o la misma figura jurídica, al aplicar solamente el delito de feminicidio, pudiera derivar en penas menores, pues no contemplaría agravantes como el secuestro y la tortura, por ejemplo.

Este trabajo pretende llenar un hueco en la falta de información y en la visibilización de los feminicidios que ocurren en el Estado de Guanajuato, México, y que van al alza. Es un trabajo exploratorio sobre el caso, que exhibe algunos resultados obtenidos por medio del análisis de la información de los periódicos locales. Olamedi (2016) afirma que no existen datos exactos sobre el número de mujeres asesinadas, las causas y motivos, así como la relación de las víctimas y los victimarios o asesinos, entre otros datos.

\section{Metodología}

Para elaborar este artículo primero tuvimos como base la Encuesta sobre las Relaciones en los Hogares (Endireh) 2006, 2011 y 2016. Esta encuesta mide la violencia hacia las mujeres a nivel nacional y por Estado federativo, y resulta pertinente analizar el caso de los feminicidios en el Estado, teniendo como contexto la violencia de género. Además de que algunos datos aquí plasmados fueron obtenidos en el proyecto "Género, violencia y marginación en escuelas de nivel básico y medio superior en el sur del Estado de Guanajuato", financiado por el Consejo Nacional de Ciencia y Tecnología (Conacyt) desde 2010. En este proyecto se encuestó a 1.521 muchachas, estudiantes de preparatoria y secundaria, y se encontró que el 20\% manifestó episodios de violencia en su contra en todos los espacios en los que ellas viven: en 
la escuela, en sus casas, en los espacios públicos. Además, 9,5\% dijo que sus madres son agredidas al interior de sus hogares. Lo que intentamos mostrar, con los datos de las Endireh y del proyecto, es que los feminicidios están directamente relacionados con la violencia que se ejerce hacia las mujeres. Es decir, en espacios donde se presentan más casos de violencia de género los feminicidios seguramente aumentarán; el problema, además, es de impunidad y de normalización de la violencia.

Los datos sobre los feminicidios tienen un problema metodológico, pues nos basamos únicamente en los casos que se muestran en los periódicos locales y estatales. Se revisó cotidianamente los diarios El Correo de Guanajuato, El Sol del Bajío, El Día y los periódicos digitales La Nota Roja y Guanajuato Sur Noticias, principalmente. Sin embargo, aun cuando no se ha revisado la documentación oficial acerca de los homicidios dolosos de las mujeres y/o feminicidios que se realizan en el Estado, consideramos que este es un primer paso para el análisis de un tema tan importante como el feminicidio, en un Estado con altas tasas de violencia hacia las mujeres. Como menciona Ascencio (2018), en la investigación en espacios peligrosos y sobre temas delicados, se debe realizar un proceso gradual, para evitar situaciones que pongan en peligro a quienes investigan. Por lo que, en este momento, la investigación solamente es hemerográfica ${ }^{3}$.

Se seleccionaron las notas periodísticas que tuvieran información sobre mujeres asesinadas en el Estado. No se consideraron casos de suicidios, accidentes $\mathrm{u}$ otros. En este momento se trata solamente de un recuento, en el cual nos basamos para reflexionar acerca de este fenómeno cada vez mayor en el Estado. Como se mencionó ya, es un trabajo exploratorio, descriptivo, de carácter también explicativo de un fenómeno en avance. 
El trabajo parte de la idea de las desigualdades estructurales entre hombres y mujeres, donde ellas se encuentran en una posición de subordinación y opresión, además de que se considera que deben estar recluidas en el ámbito privado, en actividades de reproducción y cuidado (Justo, 2005). Por lo que en el artículo el análisis se realiza desde la perspectiva de género, que es definida como "una categoría de análisis para el estudio de las construcciones culturales y sociales que se establecen entre los hombres y las mujeres que identifica lo femenino y lo masculino", entendiendo que en esta sociedad lo femenino está subordinado a lo masculino, por lo que las relaciones ente los géneros no solamente son desiguales sino que el poder lo tienen los hombres y lo ejercen sobre las mujeres. Se definen también las identidades de género. En el caso de la identidad de género de las mujeres, Rodríguez (2015) menciona tres ejes: a) maternidad; b) matrimonio o unión, y c) trabajo o profesión; estos definen lo que debe ser una mujer "buena". Cita a Lagarde para identificar otros elementos que en este sistema patriarcal definen a las mujeres, como la sexualidad para otros, la subordinación a lo masculino y la dependencia vital. Y el cuerpo de la mujer ocupado, habitado, como objeto y expropiado de ellas mismas (Rodríguez, 2015).

\section{Desarrollo y resultados}

\section{Violencia de género en Guanajuato}

Para contextualizar este trabajo se mostrarán los datos de la Encuesta sobre las Relaciones en los Hogares (Endireh, 2016), la que indica que en el Estado de Guanajuato las mujeres que han padecido violencia a lo largo de su relación de pareja alcanzan $37,5 \%$ de las mujeres encuestadas. De ellas $17,6 \%$ padece o padeció violencia física, 5,9\% violencia sexual, 18,9\% patrimonial o económica, y 34,3\% violencia emocional. 
Cuadro 1. Porcentaje de violencia hacia mujeres Guanajuatenses, de 15 años y más, según grupo de edad.

\begin{tabular}{|l|c|}
\hline Edades & Porcentaje \\
\hline 15 a 24 años & 35,7 \\
\hline 25 a 34 años & 36,9 \\
\hline 35 a 44 años & 38,9 \\
\hline 45 a 54 años & 39,3 \\
\hline 55 a 64 años & 41,3 \\
\hline 65 años y más & 36,6 \\
\hline Edad no especificada en mujeres de 15 años y más & 23,6 \\
\hline
\end{tabular}

Fuente: datos de Endireh, 2016.

Como se observa en el cuadro 1, si bien la violencia hacia las mujeres en el Estado de Guanajuato se presenta en todas las edades, incluso en niñas, hay edades en las que las mujeres pueden ser más o menos vulnerables a la violencia. Aunque las cifras no varían mucho entre los grupos etarios, los porcentajes son altos y es en el grupo de 55 a 64 años donde la violencia alcanza 41,3\%. Pareciera que las mujeres mayores son más violentadas y el grupo de 45 a 54 años alcanza porcentajes ligeramente más bajos que las mujeres de 55 a 64 años, pero en todas las edades la violencia hacia ellas está presente.

De acuerdo con los datos presentados por Ramos y Saltijeral (2011) y con la hipótesis de Izquierdo (2011), hombres con menores ingresos y con poco poder violentan más a sus parejas mujeres: Izquierdo dice que son patriarcas despojados. En este sentido, cobra relevancia lo que la Endireh (2016) muestra, aunque son datos nacionales, puesto que las mujeres que trabajan presentan mayores porcentajes de violencia de todos los tipos, a diferencia de las mujeres que solo estudian o solo buscaron trabajo. Los datos pueden verse en el cuadro 2. Este rubro nos clarifica aún más la hipótesis de que son las mujeres en proceso de empoderamiento o empoderadas (y que tienen un empleo), o las mujeres que tienen un empleo, las que más violencia padecen, como si fuera una reacción patriarcal hacia ellas para que 
regresen a lo que supuestamente debe ser una mujer. Asimismo, en el caso de los feminicidios en el Estado de Guanajuato, donde el mayor porcentaje de ellos ocurre en el llamado "corredor industrial" del Estado y donde las mujeres tienen más posibilidades de encontrar un empleo, estos datos pudieran corroborar esta hipótesis.

Los datos de violencia hacia las mujeres que trabajan o trabajaron son ligeramente mayores que las que tienen algún otro tipo de condición, que podrían ser jubiladas o que tienen una discapacidad. En este caso, la violencia física hacia estas mujeres es mayor que la que padecen o padecieron las mujeres que trabajaron.

Cuadro 2. Porcentaje de mujeres con incidentes de violencia, en pareja o alguna vez en pareja, según condición de actividad.

\begin{tabular}{|l|c|c|c|c|c|}
\hline \multicolumn{1}{|c|}{$\begin{array}{c}\text { Condición de } \\
\text { actividad }\end{array}$} & $\begin{array}{c}\text { Total con } \\
\text { incidentes } \\
\text { de violencia }\end{array}$ & Emocional & $\begin{array}{c}\text { Económica } \\
\text { o } \\
\text { patrimonial }\end{array}$ & Física & Sexual \\
\hline Trabajó & 47.4 & 43.9 & 23.6 & 19.2 & 7.8 \\
\hline $\begin{array}{l}\text { Solo es } \\
\text { estudiante }\end{array}$ & 35.5 & 34.6 & 3.4 & 5.1 & 2.3 \\
\hline $\begin{array}{l}\text { Solo se dedica a } \\
\text { quehaceres del } \\
\text { hogar }\end{array}$ & 42.1 & 37.5 & 20.9 & 18.4 & 5.8 \\
\hline $\begin{array}{l}\text { No trabajó } \\
\text { o solo buscó } \\
\text { trabajo }\end{array}$ & 42.9 & 39.3 & 19.2 & 17.3 & 6.8 \\
\hline Otra situación & 41.3 & 36.3 & 23.9 & 20.9 & 7.9 \\
\hline
\end{tabular}

Fuente: Endireh, 2016.

Nota: La suma de los tipos de violencia no coincide con el total, pues cada mujer pudo haber padecido más de uno. Otra situación incluye a las mujeres que declararon ser jubiladas, pensionadas o que tienen una limitación física o mental para trabajar. 
Gráfico 1. Prevalencia de la violencia en mujeres de 15 años o más, según tipo de violencia y año de la encuesta.

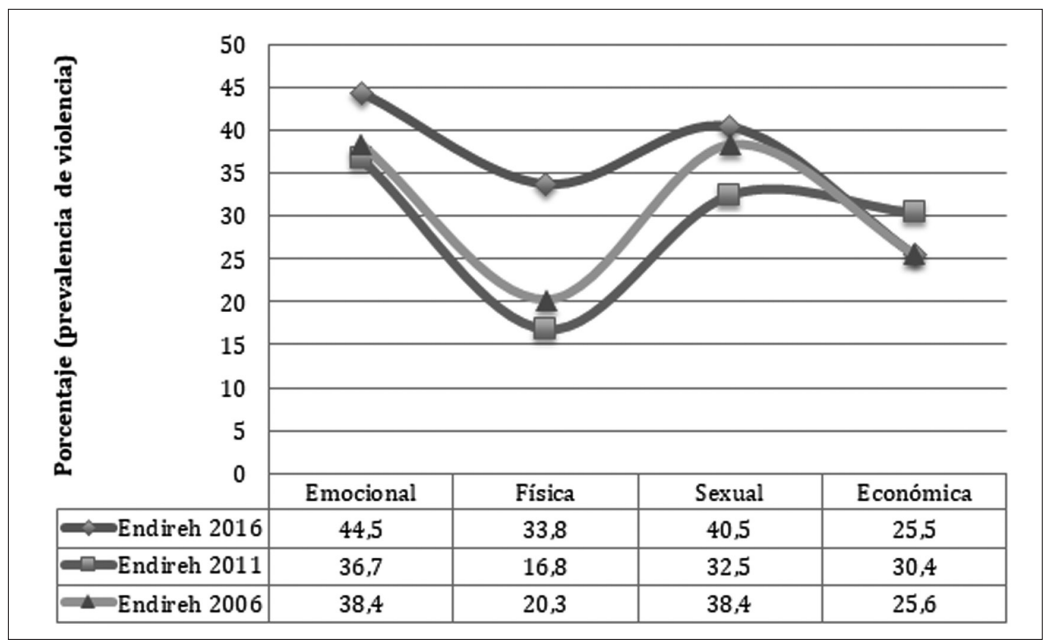

Fuente: Elaboración propia con datos de la Endireh, 2016.

El gráfico 1 muestra la prevalencia de la violencia hacia las mujeres en el Estado de Guanajuato. Es decir, el porcentaje de mujeres de 15 años y más que han sido violentadas a lo largo de su vida, por el tipo de violencia. Lo que exhibe es un aumento de los tipos de violencia en 2016. Si bien de 2006 a 2011 pareciera que la prevalencia de violencia hacia las mujeres bajó -excepto en la violencia económica, que pasó de 25,6 a 30,4\%-, de 2011 a 2016 la violencia aumentó de manera considerable, excepto en la económica, donde bajó de $30,4 \%$ a $25,5 \%$. Pero fue la violencia física la que casi se duplicó, pasando de $16,8 \%$ en 2011 a 33,8\% en 2016. Lo que es un indicativo muy fuerte pues en 2016 y 2017 los feminicidios han aumentado considerablemente.

Las mujeres de México, y las de Guanajuato especialmente, viven la violencia en diferentes espacios: en los centros escolares, en los espacios públicos, en sus trabajos e incluso en sus casas (donde se supone deberían estar más seguras).

En el sur del Estado de Guanajuato se han realizado varios estudios sobre violencia en el ámbito escolar, en espacios públi- 
cos y acerca de la violencia que las mujeres viven o han vivido en las escuelas y en sus familias a lo largo de sus vidas. Si bien son estudios que principalmente se enfocan en el Municipio de Salvatierra, el estudio sobre violencia en espacios educativos nos muestra que hay un sector de mujeres muy jóvenes que son más vulnerables a sufrir violencia en todos los espacios de su vida (Rosas y Ríos, 2013). En el estudio realizado en el sur del Estado, denominado "Género, violencia y marginación en escuelas de nivel básico y medio superior en el sur del Estado de Guanajuato", se encontró que 32\% de las estudiantes acusó violencia en sus hogares, donde los insultos y las discusiones forman parte cotidiana de sus relaciones de parentesco.

Y no solamente en los hogares o escuelas, también en espacios públicos como calles, parques, plazas y mercados, las mujeres -principalmente jóvenes- padecen agresiones por parte de los varones que frecuentan esos lugares.

En el gráfico 2 resumimos lo que viven las mujeres en el Estado (el contexto de la violencia):

Gráfico 2. El contexto de la violencia en Guanajuato.

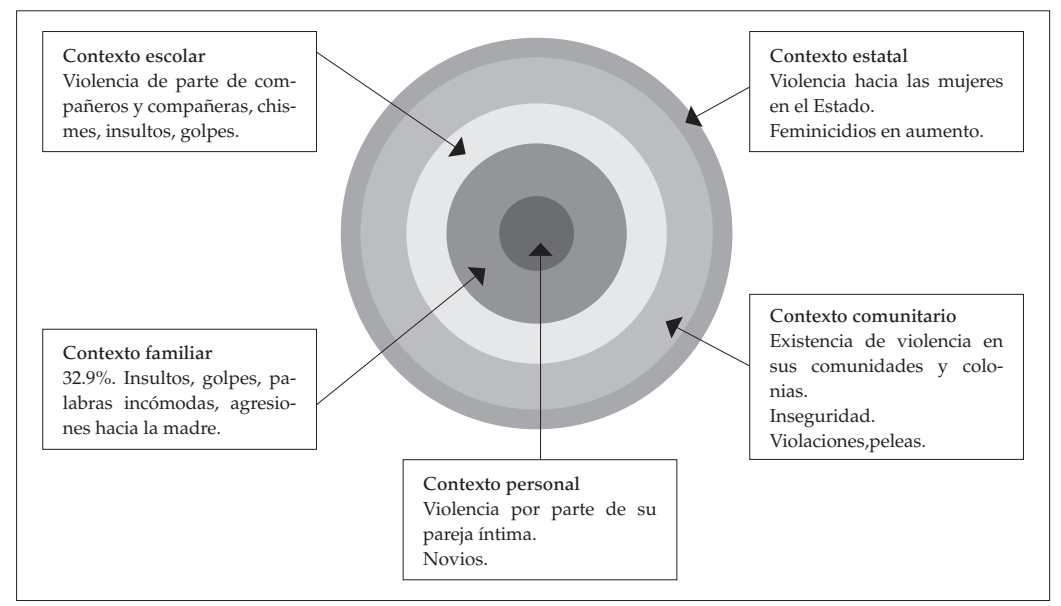

Fuente: tomado de Rosas, Valdés y Hurtado (2016, p. 29).

El gráfico 2 muestra cómo es el contexto en el que viven las mujeres guanajuatenses, caracterizado por la violencia en el ho- 
gar, con la pareja y a nivel comunitario, donde se observan escenas cada vez más violentas no solo hacia ellas sino hacia otros integrantes de la comunidad; en las escuelas, donde no solamente se habla de bullying sino de agresiones hasta por parte de profesores; la violencia de género que viven las mujeres y niñas en el Estado, y que reporta la Endireh; a todo eso se suma la normalización de la violencia y la revictimización de las víctimas al culpabilizarlas por la propia violencia que sufren; pero además la impunidad que existe, en la cual las autoridades no escuchan la voz de las mujeres y no aplican la ley. Todo esto genera es un caldo de cultivo adecuado para que se atente cada vez más contra la vida de las mujeres.

\section{Los feminicidios en el Estado de Guanajuato}

Para fines de este artículo, presentamos los datos de asesinatos de mujeres ocurridos hasta julio de 2017. Para el resto de los meses aún estamos en el trabajo de recopilación de datos. A la fecha mencionada han ocurrido 74 asesinatos de mujeres (aun no podemos afirmar que el 100\% de los casos sean feminicidios) y muchos de ellos cumplen con los supuestos que el Código Penal del Estado de Guanajuato contempla. Si bien estos casos han ocurrido en casi todos los municipios del Estado, es en la zona industrial donde más se producen. Es decir, de 74 casos aquí contabilizados, 52 han sucedido en el "corredor industrial", conformado por los municipios de León, Celaya, Apaseo el Alto, Apaseo el Grande, Irapuato, Salamanca, San Francisco del Rincón, Silao y Villagrán.

En los municipios mencionados (con excepción de Cortázar, donde hasta la fecha mencionada aún no había ocurrido ningún feminicidio) acaecieron 70,3\% de los asesinatos femeninos en el Estado, y León ocupa el primer lugar, seguido por Celaya: 52 asesinatos de los 74 ocurridos pasaron en esa zona. La mayor parte (27) por arma de fuego ${ }^{4}$.

4 En esta región del corredor industrial (especialmente en los municipios de Celaya, Apaseo el Grande, Apaseo el Alto y Villagrán) es donde se perpetra con 
En el norte del Estado hubo seis asesinatos de féminas, uno en cada uno se los siguientes municipios: San Felipe, San Miguel de Allende, San Luis de la Paz y Guanajuato, mientras que en el de Santa Cruz de Juventino Rosas ocurrieron dos.

En el sur del Estado ocurrieron también estos episodios violentos, y solamente en dos casos se conoce al asesino: uno en Santiago Maravatío, donde un hombre asesinó a su pareja, y otro en Abasolo, donde el hijo mató a su madre.

Es el arma de fuego la forma más utilizada en el Estado para matar a las mujeres, sobre todo si los asesinos aparecen como desconocidos, pero cuando el asesino se conoce esta situación cambia, como lo vemos en la gráfica de abajo. Solamente se presentan los casos donde se conoce la identidad del asesino y que es o fue muy cercano a la víctima.

Gráfico 3. Asesinatos de mujeres en Guanajuato, por tipo y asesino conocido,

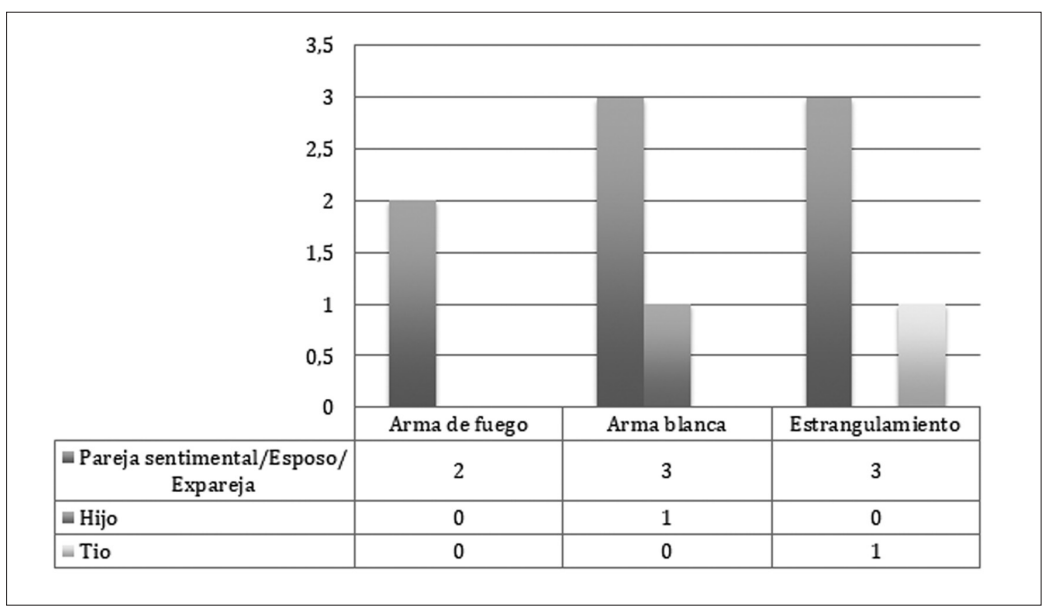

Fuente: elaboración propia.

mayor frecuencia el robo de combustible (llamado coloquialmente "huachicoleo") y donde los asesinatos van en aumento debido a este ilícito y al tráfico de drogas. 
Si bien el arma de fuego sigue siendo el medio más usado para matar a las mujeres, los hombres que fueron parejas o que aún eran sus parejas sentimentales o esposos las mataron usando cuchillos y machetes, en tres casos, y en otros tres las estrangularon. Es decir parecen adoptar formas más crueles de matar a las mujeres que fueron muy cercanas a ellos.

Mientras, como se observa en el gráfico 4, cuando se desconoce la identidad de los asesinos o no son cercanos (en este caso es un vecino), la manera más usada es asesinarlas con arma de fuego, con $64 \%$ de casos (equivalente a 41 mujeres); son formas más rápidas de asesinarlas. Hay dos casos de asesinatos con armas blancas, uno a golpes, otro a pedradas, otro con quemaduras, seis estranguladas. Es decir, aun cuando obviamente mataron a la mayoría de las mujeres con disparos, los casos en los que las matan con mayor dolor y saña se producen si tienen vínculos muy cercanos con los asesinos.

Gráfico 4. Asesinatos de mujeres en Guanajuato, por tipo y asesino desconocido.

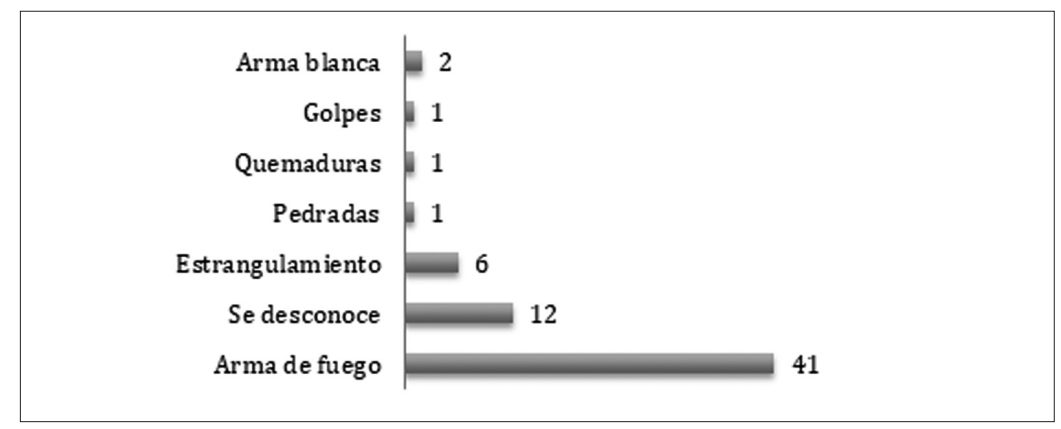

Fuente: elaboración propia.

Los datos que se presentan son hasta julio de 2017, pero los asesinatos continuaron y alcanzaron cifras mayores que en 2016, cuando se contabilizaron 106 mujeres asesinadas. Se presentaron patrones similares, el "corredor industrial" con más casos, León en primer lugar y Celaya en un nada honroso segundo lugar. 
Como se dijo, pareciera que si hay vínculos cercanos con las víctimas los asesinatos son más crueles: En un caso de 2016, a una mujer su pareja sentimental la asesinó pero infringiéndole mucha violencia pues abusó sexualmente de ella al momento de matarla, le realizó al cadáver varias lesiones externas, una de las cuales afectó la arteria carótida, la yugular y seccionó la tráquea. Luego de asesinarla, la quemó. En Celaya una mujer trans fue asesinada, encontraron su cuerpo calcinado, en este caso se desconoce al asesino, pero la violencia fue mayúscula pues pareciera que quisieron borrar rastros o huellas de quién había sido esta mujer trans. En ambos casos la misoginia se encuentra presente.

Quizás los casos en los que se conoce a los asesinos, donde pareciera que a las muertes las antecedió violencia extrema (dada las condiciones en que se encontraron los cadáveres) responda a lo que Ramos y Saltijeral (2011) llamaron "terrorismo íntimo": violencia ejercida por hombres despojados o disminuidos, que usan las acciones violentas (incluso el asesinato) para autorreafirmarse, cosa que tal vez no puedan hacer hacia al exterior.

¿Cuál es el problema con los feminicidios?, además de que privan a las mujeres de su vida, que se supone sería lo más grave. La gravedad es aún mayor: es el machismo imperante en la sociedad, que no solo vulnera los derechos de las mujeres, sino que culpa a las mismas mujeres de su muerte. En una sociedad en la que se naturaliza la violencia contra las mujeres, donde se valora menos lo femenino, es lógico que la culpa de los sucesos violentos hacia ellas recaiga en ellas mismas.

En el caso de una joven encontrada en un hotel en León, a donde acudió con un desconocido, como relata el periódico, las personas en el Estado solamente criticaron que fue con un desconocido a un hotel teniendo ella novio (lo normal era que fuera con el novio, o mejor que nunca fuera a ningún hotel). La culpa de que la asesinaran recayó en ella, pues fue juzgada por sus acciones, algunas de las opiniones son las siguientes: 
"X [por] facilota q hacia en un hotel con un desconocido si tenía novio".

"Por andar de puta. Lástima, por una tarde de pasión terminó muerta".

"Si hubiera unaxhava [chava] buen [bien] portada y de valores no le hubiera pasado eso tienes toda la razón nadie tiene derecho a quitarle la vida pero neta q pen. $Q$ se can con cual quieres cabrón teniendo novio y a demás un desconocido q esperan krs oase si el país está cono esta es ata jodido por la delincuencia". "Inocente?".

(Las opiniones son copia textual de la página web del periódico).

En este caso quienes opinan juzgan a la chica, justificando su asesinato porque la consideran una mujer "fácil," puta o que no tiene los valores adecuados a una "buena o respetable mujer." Es decir, hay casos (o muchos casos de feminicidio, violencia sexual y de otros tipos) que la opinión pública juzga no sancionables y los vuelve impunes.

No solamente se normaliza la violencia hacia las mujeres, se justifica el asesinato, incluso hay violencia moral, que es definida como "el conjunto de mecanismos legitimados por la costumbre para garantizar el mantenimiento de los estatus relativos entre los términos de género. Estos mecanismos de preservación de sistemas de estatus operan también en el control de la permanencia de jerarquías en otros órdenes, como el racial, el étnico, el de clase, el regional y el nacional" (Segato, 2010, pp. 105-106).

$Y$ en el caso que se menciona es claro que se sanciona moralmente a la víctima, ya que no se portó como una joven de "bien", con los supuestos valores morales que se esperan de ella. Es decir, se fue con otro y no con "su" novio. Por lo que el castigo por no ser y portarse como la sociedad dicta a una mujer se castiga con la muerte.

La misma Segato indica que la violencia es una forma de preservación del sistema, en este caso patriarcal, porque no solo 
los cuerpos de las mujeres padecen la violencia que las llevó a la muerte, sino que incluso más allá siguen siendo violentadas al juzgar su conducta y por tanto responsabilizarlas de su asesinato. "La violencia moral, por su invisibilidad y capilaridad, es la forma corriente y eficaz de subordinación y opresión femenina, socialmente aceptada y validada" (Segato, 2010, p. 113).

Lo anterior provoca una gran impunidad para resolver los feminicidios. Hay pocos casos resueltos, "pésimas investigaciones y movilización policiaca; averiguaciones mal integradas; ministerios públicos dolosos y machistas que desatienden las denuncias de las víctimas, y jueces misóginos para quienes la vida de las mujeres es secundaria" (Lagarde, 2006, pp. 18-19). Y si existe algún asomo de vínculo probable con el crimen organizado, mejor ni se investiga. Lo saben los hombres, que asesinan mujeres y tratan de imitar los crímenes del narcotráfico. Por ejemplo, el feminicidio sucedido en el Motel Ecko en Salvatierra, Guanajuato, en el que el novio asesinó a la joven ahorcándola con el cable del ventilador y en el espejo del baño dejó una nota, escrita con lápiz labial, diciendo que era un ajuste de cuentas. Por fortuna, la joven había enviado un mensaje de texto a una amiga informándole dónde y con quién estaba, y eso ayudó a esclarecer el crimen, que hubiera quedado impune si solamente se guían por el mensaje en el espejo.

Nussbaum afirma que las mujeres son ciudadanas de segunda clase, porque "carecen de un apoyo esencial para llevar una vida plenamente humana. Esta falta de apoyo se debe al solo hecho de ser mujeres" (Nussbaum, 2002, p. 31), y ello las pone en gran riesgo de que sus derechos sean vulnerados, incluyendo el derecho a una vida libre de violencia y el derecho a la vida.

Los feminicidios pueden tener varios significados, uno el mencionado por Izquierdo (2011), en el que los homicidas son patriarcas despojados. Ravelo (2005) en cambio nos dice que puede existir crisis de masculinidad, además de la de pareja, en la que ellos, al no cumplir con el papel que les toca como proveedores, 
recurren al asesinato de sus esposas y/o hijos o hijas. Pero también pueden ser formas simbólicas de castigo (como el asesinato en el motel El Mexicano, en la ciudad de Salvatierra, donde un hombre asesinó a una mujer porque supuestamente ella intentó robarle), y de reafirmación del poder masculino. Ravelo (2005) indica que en los crímenes contra las mujeres hay sesgos misóginos y patriarcales, como los celos irracionales y la idea de que los hombres son poseedores de los cuerpos femeninos. Tanto propios como extraños se creen en la libertad de poseer los cuerpos de las mujeres, aun contra su voluntad y con menor o mayor violencia, ya que la cultura les ha conferido el derecho sobre ellas y las consideran a todas como de su propiedad.

Las mujeres y niñas asesinadas, dice Marcela Lagarde, tienen "alto grado de inseguridad, vulnerabilidad vital y nula protección social e institucional, en zonas de devastación social donde predominan la inseguridad, el delito, una convivencia marcada por la ilegalidad, los poderes fácticos, el desbordamiento de las instituciones y la ruptura del Estado de derecho" (Lagarde, 2006, p. 23). Tanto Celaya como León han sido mencionadas como dos de las ciudades más inseguras del Estado, donde día con día se cometen infinidad de delitos. En León no hay un día en el que la prensa no de cuenta de uno o varios asesinatos.

El "corredor industrial" del Estado se caracteriza por una concentración importante de industria, de fuentes de empleo y, se supone, de mayor bienestar. Entonces, quizás los asesinatos sucedan en esta zona porque las mujeres ya tienen acceso a empleo, a servicios educativos, y quizás estén en camino de empoderamiento, lo que puede provocar mayor violencia hacia ellas o que sean asesinadas. Pero Lagarde (2006) afirma que es en zonas con alto grado de vulnerabilidad, inseguridad y nula protección social donde ocurren mayormente los asesinatos de mujeres, "en zonas de devastación social donde predominan la inseguridad, el delito, una convivencia marcada por la ilegalidad, los poderes fácticos, el desbordamiento de las instituciones y la ruptura del estado de derecho" (Lagarde, 2006, p. 23). Es lo que puede estar 
sucediendo en el "corredor industrial", principalmente en León y Celaya, ciudades con altos índices de criminalidad y donde el crimen organizado entabla cruentas y sangrientas batallas. La misma autora señala que en lugares con altos índices de asesinatos de hombres aumenta el número de feminicidios, cosa que parece ocurrir en este estado de la República mexicana.

La violencia de género es alta en el Estado de Guanajuato. Lagarde (2006) afirma que los asesinatos de mujeres coinciden con una enorme violencia hacia ellas, de todo tipo, numerosas formas de exclusiones, pobreza, falta de oportunidades, trata, desapariciones, etcétera. Lo que ocurre ahora en el Estado se agrava cada vez más, pareciera que no se alcanzan los niveles deseados de justicia. La impunidad está a sus anchas. Hay numerosas desapariciones de jovencitas en el Estado, raptos a la luz del día ${ }^{5}$.

A veces nos preguntamos ¿por qué suceden estas cosas? ¿Qué motivos tendrían los asesinos? La teoría nos indica que el sistema patriarcal en el que vivimos es el que propicia las desigualdades entre hombres y mujeres; genera que lo masculino sea más valioso y lo femenino no se valore, y hace que estas cosas sucedan. Es decir, hay una ideología en la que lo femenino es subestimado y debe ser controlado por los hombres; entonces, ellos hacen lo necesario para controlar a "sus mujeres." En una noticia de abril de este año, en Juventino Rosas, un hombre apuñaló a su exesposa porque ella no quería regresar a vivir con él. Discuten por el regreso, ella se niega y él la apuñala, demostrando que lo que importa es lo que él piense, lo que él quiera, y la mujer es "suya" y debe hacer lo que él diga; como no lo hizo, la apunala. Afortunadamente la mujer vivió para contarlo.

Una joven fue raptada por unos hombres a plena luz del día, frente a testigos. Nadie pudo (o quiso) hacer algo; se denunció el hecho pero, desgraciadamente, el cadáver de la joven se encontró días después. Lagarde (2006) dice que el horario es limitado para las mujeres. Pero ahora ni las horas del día, ni caminar entre mucha gente, ni ir acompañada es seguro, como pasó con la joven. Iba con una familiar, había más personas (no estaba en un lugar solitario), era mediodía. No hubo forma de salvarla. 
Las notas periodísticas sobre violencia hacia las mujeres son escasas, no porque no existan, sino porque las que aparecen son las más brutales. En San Francisco del Rincón una mujer denuncia a su pareja: iban caminando y de repente él le empieza a pegar y le hace una herida en la cabeza.

En otra nota, un hombre de 29 años discute con su esposa de 44 y la apuñala. Él fue acusado de homicidio en grado de tentativa.

Lo que se intenta decir con esto es que en este Estado de la República mexicana existen altos índices de violencia hacia las mujeres, de todo tipo: sexual, psicológica, económica, patrimonial, simbólica, incluyendo violencia extrema (como los casos arriba señalados), y que esas violencias van escalando y producen los asesinatos de las mujeres a manos de sus parejas, novios, exnovios, esposos, exesposos, desconocidos y parientes. Las evidencias encontradas en el trabajo de campo del proyecto "Género, violencia y marginación en escuelas de nivel básico y medio superior en el sur del Estado de Guanajuato" remiten a mujeres jóvenes que padecen violencia en distintos ámbitos; mujeres adultas que desde niñas la padecieron y la siguen padeciendo; falta de políticas claras que ataquen este problema creciente; misoginia, ignorancia y prejuicios por parte de quienes deberían impartir justicia por estos delitos y que, sin embargo, no lo hacen porque desdeñan la palabra de las mujeres; ignorancia deliberada de los casos de mujeres asesinadas, minimizando el número de ellas y las circunstancias. Además, instituciones de profundo arraigo conservador, como la Iglesia católica, cuyos representantes culpabilizan a las mujeres por la violencia que padecen y por los asesinatos, que las juzgan por sus vidas y ropas y no juzgan ni mencionan a los perpetradores. Este es el ámbito que permite, entre otras desigualdades, que la violencia crezca y que junto con ella aumenten los feminicidios en el Estado.

Por otra parte, en ocasiones hay un mal manejo de la información en los periódicos, ya que se emiten juicios que justifican los 
asesinatos. Por ejemplo, en uno de ellos una mujer fue asesinada por su ex novio, la dejó semidesnuda en el estacionamiento de un centro comercial y envuelta en una cobija. De este caso la nota periodística afirma que la joven asistía con frecuencia a bares y este comentario no tiene que ver directamente con el asesinato, pero sí con la conducta de ella y puede justificar (y de hecho lo hace) ante la opinión pública el asesinato. Este tipo de comentarios sobre la conducta de las víctimas nos indica que solamente las mujeres que tienen conductas no "adecuadas socialmente" pueden ser asesinadas, y esto no es verdad.

Analizando los casos, la impunidad con la que se presentan los feminicidios en Guanajuato, donde las autoridades descartan que sean feminicidios, las pocas acciones gubernamentales para que las mujeres guanajuatenses vivan una vida libre de violencia, los prejuicios de género con los que actúan las autoridades, los roles de género tradicionales, entre otros, hacen que las condiciones sociales en el Estado no sean propicias para resguardar la integridad y vida de las mujeres, ya que "la vida exige apoyo y unas condiciones capacitadoras para poder ser una vida "vivible" (Butler, 2011, p. 40).

Hay que repensar el derecho a la vida, ahí donde las condiciones no son precisamente las adecuadas para vivirla, donde implica entonces asegurar las mínimas condiciones para que las vidas puedan dejar de ser tan precarias. Butler (2011) habla de apoyos, tales como la comida, el cobijo, el trabajo, la atención sanitaria, la educación, el derecho a la movilidad y a la expresión, y a la protección contra los daños y contra la opresión. Muchas de las mujeres violentadas y las asesinadas no tuvieron estas condiciones y la protección contra los daños y opresión no las alcanza.

\section{Conclusiones}

En México -y en el Estado de Guanajuato, en este caso- tenemos leyes que resguardan los derechos humanos de las mujeres, el derecho a la vida y a vivirla sin violencia, por ejemplo. Pero 
estos derechos no solo se deben reconocer jurídicamente, sino ser sancionados socialmente y no solamente ser letra que no se cumple; como en el Estado de Guanajuato, donde se reconoce el derecho de las mujeres a vivir una vida libre de violencia, pero no existen las condiciones sociales para su aplicación real, por lo que se infiere que las mujeres guanajuatenses no tienen el acceso a un derecho fundamental: el de la vida, y sus vidas se tornan aun más precarias, ya que resulta peligroso vivir en este Estado de la República mexicana.

Para garantizar la seguridad de las mujeres hacen falta políticas y acciones que vayan más allá de pronunciarse algunos días al año en contra de la violencia de género, más allá de anuncios espectaculares, pues en los municipios, aun cuando existan institutos municipales de la mujer, estos carecen de la infraestructura necesaria para apoyar a las mujeres, incluso no tienen capacitación y a veces se enfrentan a la oposición de los presidentes municipales. Las mujeres muchas veces no saben que estos institutos existen, y si todas los conocieran seguramente rebasarían las posibilidades de ayuda.

Presentamos solamente datos hasta julio de 2017, pero aún falta no solamente visibilizar estos crímenes, sino clasificarlos a partir del Código Penal y de lo que indica la literatura. Sobre todo ahora que en el Estado hay mucha violencia originada por el narcotráfico y para evitar aun más impunidad, lo que implicaría no solamente un trabajo académico, sino jurídico y de reconocimiento de los derechos de las mujeres.

La mayor parte de los asesinatos de mujeres ocurridos a julio de 2017 han sido perpetrados por desconocidos y en su mayoría utilizando un arma de fuego. En los casos de asesinos conocidos, fueron los esposos, exesposos y parejas quienes asesinaron a las mujeres, además de un tío y un hijo. Pero en estos últimos casos pareciera que se quiso infligir más dolor a las víctimas, pues se utilizaron medios más crueles para asesinarlas, como el uso de machetes y cuchillos y el estrangulamiento. Probablemente en el 
caso de hombres conocidos la crueldad es mayor, quizás por un odio más acentuado hacia las mujeres que compartieron su vida. En estos casos, Cruz (2011) también nos habla del "terrorismo fálico" que se ejerce sobre el cuerpo de las mujeres, cuyo fin es el goce sexual y la posesión de ellas para posteriormente desechar los cuerpos. En estos casos que la autora estudia en Ciudad Juárez resalta la extrema violencia que se ejerció sobre ellas y les produjo la muerte.

En 2016 y 2017 los casos se concentran en la zona de mayor pujanza económica e industrial, y también en la más poblada del Estado. Lo que puede indicar una mayor violencia hacia las mujeres con algunos indicios de empoderamiento o que quisieran trascender más allá del rol tradicional patriarcal que la sociedad les impone.

Las explicaciones sobre los feminicidios pueden ser varias, pueden ser hombres en una crisis de masculinidad (patriarcas despojados), en la que lo único que pueden controlar es el cuerpo de "sus" mujeres, y que a lo largo de la relación de pareja predominó el terrorismo íntimo o patriarcal en las relaciones. También puede ser que los feminicidios sean formas de enseñanza a las otras mujeres que están vivas, de lo que puede pasarles si no se comportan "adecuadamente", según los roles tradicionales de género que aún imperan. Pero aun cuando las mujeres se decidieran a dedicar sus vidas al trabajo reproductivo, muchas de ellas son asesinadas en sus casas, por sus esposos o exparejas, por lo que tampoco los hogares son un lugar seguro para las mujeres y niñas.

En una sociedad como la guanajuatense, con roles muy tradicionales de género, donde la cultura patriarcal indica los comportamientos, trabajos y acciones adecuadas a hombres y mujeres, la violencia de género alcanza niveles altos, pero se normaliza y se piensa que como siempre ha ocurrido así no se puede cambiar la realidad. Al ocurrir esta situación, los feminicidios son justificados por la sociedad, porque se culpa a las mujeres, ocurren en una so- 
ciedad en la que las leyes no se cumplen, los funcionarios no cumplen con su trabajo y emiten una serie de prejuicios de género que obstaculizan el acceso de las mujeres a la justicia; ocurren en un Estado controlado ya por las mafias del narcotráfico (donde a mayo de 2018 los asesinatos de mujeres sumaban más de 100 casos); donde se minimizan los casos de violencia de género hacia las niñas y mujeres; donde prima la impunidad también. No es extraño que los asesinatos de mujeres, ahora que el Estado de Guanajuato es uno de los más violentos en el país, se hayan incrementado a niveles que no se había observado en años anteriores.

Finalmente, quiero aclarar que si usamos el término "tradición" en estos casos nos referimos más a la tradición de la violencia que las mujeres guanajuatenses sufren durante su vida, cuando niñas, adolescentes, mujeres jóvenes y en la vejez. Que no solamente sufren las mujeres actuales sino sus madres, abuelas y que en muchos casos ocurrirá en sus hijas. Por eso ellas las enseñan a ser "buenas" mujeres, a portarse bien, con el fin de evitar lo que en el fondo saben que no se puede: la violencia hacia ellas y en algunos casos su asesinato.

\section{Referencias}

Ascencio, C. (2018). Pactando con el diablo: problemas metodológicos y éticos de investigación en contextos violentos. Acta Sociológica, 75, 87-111.

Arteaga, N. (Coordinador). (2010). "Por eso la maté." Una aproximación sociocultural a la violencia contra las mujeres. México: Miguel Ángel Porrua, Universidad Autónoma del Estado de México.

Arteaga, N. y Valdés, J. (2010). Contextos socioculturales de los feminicidios en el Estado de México: nuevas subjetividades femeninas. Revista Mexicana de Sociología, 72 (1), 5-35. Recuperado el 16 de noviembre de 2017, de http:/ /www.scielo. org. $\mathrm{mx} /$ scielo.php?script=sci_arttext\&pid=S018825032010000100001\&lng=es\&tlng=es.

Blanco, J. (2009). Rostros visible de la violencia invisible. Violencia simbólica que sostiene el patriarcado. Revista Venezolana de 
Estudios de la Mujer, 14 (32), 63-70. Recuperado en 23 de noviembre de 2017, de https://dialnet.unirioja.es/ejemplar/320886.

Bodelón, E. (2009). Feminismo y derecho: mujeres que van más allá de lo jurídico, en G. Nicolás y E. Bodelón (Comps.), Género y dominación. Críticas feministas al derecho y al poder. España: Anthropos.

Butler, J. (2011). Marcos de guerra: las vidas lloradas. España: Paidós.

Castañeda S., Ravelo, M. y Pérez, T. (2013). Feminicidio y violencia en México: omisiones del estado y exigencia civil de justicia. Revista de Ciencias Sociales y Humanidades, (74), 11-39. Recuperado el 16 de noviembre de 2017, de file:/ / Users / rociorosasvargas / Downloads / Dialnet-FeminicidioYViolenciaDeGeneroEnMexico-4692151.pdf

Cruz, S. (2011). Terrorismo fálico y violencia masculina: ejercicios de poder en el feminicidio de Ciudad Juárez, en M. G. Huacuz Elías (Coord.), La bifurcación del caos. Reflexiones interdisciplinarias sobre violencia falocéntrica. México: UAM-X.

Encuesta Nacional sobre la Dinámica de las Relaciones en los Hogares (Endireh). (2016). Recuperada de http://www.beta. inegi.org. $\mathrm{mx} /$ proyectos/enchogares/especiales/endireh/2016/.

Freyermuth, G. (2003). Las mujeres de humo. Morir en Chenaló. Género, etnia y generación, factores constitutivos del riesgo durante la maternidad. México: CIESAS, Instituto Nacional de las mujeres, Comité por una maternidad voluntaria y sin riesgos en Chiapas y Porrúa editores.

Huacuz, M. G. (Coord.) (2011). La bifurcación del caos. Reflexiones interdisciplinarias sobre violencia falocéntrica. México: UAM-X.

Izquierdo, M. J. (2011). La estructura social como facilitadora del maltrato, en M. G. Huacuz Elías (Coord.), La bifurcación del caos. Reflexiones interdisciplinarias sobre violencia falocéntrica. México: UAM-X.

Justo, C. (2005). El feminismo frente a las nuevas tecnologías de la información y la comunicación, en N. Blazquez y J. Flores (Eds.) Ciencia, Tecnología y género en Iberoamérica. México: UNAM, UNIFEM, Plaza y Valdés, Centro de Estudios Interdisciplinarios en Ciencias y Humanidades.

Lagarde, M. (2006). Introducción. Por la vida y la libertad de las mu- 
jeres. Fin al feminicidio, en D. E. Russell y R. A. Harmes (Eds.), Feminicidio: una perspectiva global (pp. 15-42). México: Centro de Investigaciones Interdisciplinarias en Ciencias y Humanidades, UNAM.

Lagarde, M. (2006a). Presentación, en D. E. Russell y R. A. Harmes (Eds.), Feminicidio: una perspectiva global. México: Centro de Investigaciones Interdisciplinarias en Ciencias y Humanidades, UNAM.

Las Libres. (2009). Diagnóstico situacional de la violencia social y de género en el Estado de Guanajuato. México: Indesol, Las Libres.

Ley General de Acceso de las Mujeres a una Vida Libre de Violencia. Publicada en el Diario oficial de la Federación, 1 de febrero de 2007. Recuperado el 23 de noviembre de 2017, de http:/ / www.dof.gob.mx/nota_detalle.php?codigo $=4961209 \& f e c$ ha $=01 / 02 / 2007$.

Marroni, M. G. (2004). Violencia de género y experiencias migratorias. La percepción de los migrantes y sus familiares en las comunidades rurales de origen, en M. Torres (Comp.), Violencia contra las mujeres en contextos urbanos y rurales. México: El Colegio de México, Programa Interdisciplinario de Estudios de Género.

Monárrez, J. E. (2011). El continuo de la lucha del feminismo contra la violencia o morir en un espacio globalizado transfronterizo: teoría y práctica del movimiento anti-feminicida en Ciudad Juárez, en M. G. Huacuz Elías (Coord.), La bifurcación del caos. Reflexiones interdisciplinarias sobre violencia falocéntrica. México: UAM-X.

Muñiz, E. (2011). El cuerpo y sus circunstancias. Una mirada cultural de la vida sexual, en M. G. Huacuz Elías (Coord.), La bifurcación del caos. Reflexiones interdisciplinarias sobre violencia falocéntrica. México: UAM-X.

Nussbaum, M. C. (2002). Las mujeres y el desarrollo humano. España: Herder.

Pérez, J. (2010). Del derecho al hecho. Leyes sobre violencia en el Estado de Guanajuato. México: Indesol, Vereda Thermis.

Ramos, L. y Saltijeral, M. T. (2011). Violencia episódica, violencia cruza$\mathrm{da}$, terrorismo íntimo... ¿vale la pena diferenciar en subtipos la violencia física contra las mujeres en el ámbito de la pareja?, en M. G. Huacuz Elías (Coord.), La bifurcación del 
caos. Reflexiones interdisciplinarias sobre violencia falocéntrica. México: UAM-X.

Ravelo, P. (2005). La costumbre de matar: proliferación de la violencia en Ciudad Juárez, Chihuahua, México. Nueva antropología, 20 (65), 149-166. Recuperado en 04 de junio de 2018, de http://www.scielo.org.mx/scielo.php?script=sci_ arttext\&pid=S0185-06362005000200009\&lng=es\&tlng=es.

Rodríguez, A. (2015). Género, identidad y violencia de género, en J. Del C. Chávez Carapia, Perspectiva de género una mirada de universitarias. México: UNAM.

Rosas, R. y Ríos, M. (Coord.) (2013). Género y diversidad cultural. México: Universidad de Guanajuato y Ed. Altres Costa Amic.

Rosas, R., Valdés, A. y Hurtado, T. (2016). El contexto sí importa. Violencia de género hacia las estudiantes en escuelas secundarias y preparatorias en Guanajuato. La Manzana de la Discordia, 8 (2). 17-30, Recuperado de http://manzanadiscordia. univalle.edu.co/index.php/la_manzana_de_la_discor$\mathrm{dia} /$ article/view/1538

Russel, D. E. H. (2006). Definición de feminicidio y conceptos relacionados, en D. E. Russell y R. A. Harmes (Eds.), Feminicidio: una perspectiva global. México: Centro de Investigaciones Interdisciplinarias en Ciencias y Humanidades, UNAM.

Russel, D. E. (2006). Feminicidio: la "solución final" de algunos hombres para las mujeres, en D. E. Russell y R. A. Harmes (Eds.), Feminicidio: una perspectiva global (pp. 15-42). México: Centro de Investigaciones Interdisciplinarias en Ciencias y Humanidades, UNAM.

Russel, D. E. (2006). Introducción: las políticas del feminicidio, en D. E. Russell y R. A. Harmes (Eds.), Feminicidio: una perspectiva global (pp. 15-42). México: Centro de Investigaciones Interdisciplinarias en Ciencias y Humanidades, UNAM.

Segato, R. (2010). Las estructuras elementales de la violencia. Ensayos sobre género entre la antropología, el psicoanálisis y los derechos humanos. Argentina: Prometeo Libros.

Segato, R. (2006). ¿Qué es un feminicidio? Notas para un debate emergente. Serie antropología, (401) Brasilia. Recuperado en 23 de noviembre de 2017, de http://cuentaconmigo.org.mx/ articulos/segato.pdf 